\title{
Zum Erwerb der interkulturellen Kompetenz in Zeiten der Digitalisierung
}

\author{
Elif ERDOĞAN*
}

\section{ZUSAMMENFASUNG}

Eine Fremdsprache zu beherrschen bedeutet nicht nur über ein ausreichendes, sprachliches Wissen zu verfügen, es hat immer etwas mit der Begegnung der Zielkultur zu tun. Infolgedessen ist das wesentliche Ziel des Fremdsprachenunterrichts, Zugang zu einer anderen Kultur zu suchen und den Fremdsprachenlerner darauf aufmerksam zu machen, dass in einer anderen Kultur; Wörter, Sprechtätigkeiten usw. einen anderen Sinngehalt haben können.

Doch die Fremdsprachenlerner, die in zielsprachenfernen Regionen leben, haben oft keine Möglichkeit außer einem Text in einem Lehrbuch, oder außer einem Video, in denen Gespräche künstlich gesteuert sind, eine Innenansicht der Zielkultur zu bekommen. Die Zielkultur ist weder in der Klasse noch in der persönlichen Umgebung vorhanden und wird im Fremdsprachenunterricht oft nur über Medien vermittelt, die meist keine Authentizität haben. Aus diesem Grund reichen Lehrbücher und Lehrkräfte allein nicht aus, um dieses relevante Ziel zu erreichen. Im Zeitalter der Digitalisierung ist es jedoch nicht mehr unwahrscheinlich dieses Ziel zu realisieren.

In diesem Beitrag wird dieses Thema näher bearbeitet und mithilfe von einer Umfrage, an der die Studierenden im Studienfach Germanistik der Universität Selcuk teilgenommen haben, versucht herauszufinden, ob die Fremdsprachenlerner durch soziale Medien landeskundliches Wissen über die Zielkultur erwerben können und welche Rolle die sozialen Medien beim landeskundlichen Lernen spielen.

Schlüsselwörter: Kultur, Interkulturalität, Interkulturelle Kompetenz, Landeskunde, Soziale Medien.

\section{Sosyal Medyanın Kültürlerarası Yetinin Edinilmesi Üzerindeki Rolü}

ÖZ

Farklı kültürlere mensup insanlar arasında başarılı bir iletişim kurabilmek için sadece o dilin gramer yapısını ya da sözdizimi kurallarını bilmek yeterli değildir, aynı zamanda hedef dilin kültürünü de bilmek gerekir. Bu nedenle yabancı dil derslerinin amacı öğrencilere sadece dilbilgisi konularını aktarmak değıl, hedef dilin farklı bir kültür yapısına sahip olduğunu ve bu kültürün özelliklerinin; kelimelerin veya davranışların hedef kültürde farklı anlamları olabileceğini öğretmek olmalıdır.

İçinde yaşadığımız dijitalleșme çağında neredeyse artık herkes sosyal medyayı aktif bir șekilde kullanmaktadır. Bunun olumsuz yönleri olduğu gibi yabancı dil olarak Almanca öğrenenler için olumlu etkileri de vardır. Örneğin hedef dilin kullanıldığı ülkeden ve kültüründen uzakta yaşayan yabancı dil öğrencileri, bir Almanın günlük hayatını yansıttı̆̆ Instagram'daki hikayelerinden ya da YouTube'deki bir video kanalından hedef dilin kültürünü tanıma şansını yakalar. Kısacası sosyal medya aracıllı̆ıyla öğrenciler, yabancı dil derslerinin en önemli hedefi olan farklı kültürleri tanıma imkanını bulur.

Bu çalısmanın amacı Selçuk Üniversitesi, Edebiyat Fakültesi, Alman Dili ve Edebiyatı bölümü öğrencilerinin katıldığı bir anket yardımıyla, öğrencilerin sosyal medya aracılığıyla hedef dilin ülke bilgisi hakkında bilgi edinip edinemediklerini ortaya çıkarmak ve sosyal medyanın ülke bilgisi öğrenimindeki rolünü belirlemektir.

Anahtar Kelimeler: Kültür, Kültürlerarasılık, Kültürlerarası Yeti, Ülke Bilgisi, Sosyal Medya.

\section{Einleitung}

„Fremdsprachenlernen beißt: Zugang zu einer anderen Kultur suchen."

(Krumm, 1994)

In den ersten Jahren des Deutsch als Fremdsprachenunterrichts konzentrierte man sich vielmehr auf sprachliche und literarische Inhalte. Mit der zunehmenden Politisierung und Soziologisierung der Gesellschaft nach 1968, kann eine landeskundliche Wende des Fremdsprachenunterrichts aufgezeichnet

* Dr. Öğr. Üyesi, Selçuk Üniversitesi, eliferdogan@selcuk.edu.tr

Makalenin Gönderim Tarihi: 06.04.2021; Makalenin Kabul Tarihi: 14.07.2021 
werden (Reimann, 2015, S. 1). Die Beherrschung einer fremden Sprache ist demnach nicht mehr mit einem ausreichenden, sprachlichen Wissen möglich. Um in interkulturellen Situationen richtig und angemessen kommunizieren zu können, spielt die interkulturelle Kompetenz, ein übergeordnetes Ziel interkultureller Bildung, eine sehr wichtige Rolle.

Sie wird unter anderem als eine Empathiefähigkeit verstanden, sich in Menschen aus anderen Kulturen hineinzuversetzen und zu versuchen, ihr Verhalten und ihre Gedanken zu verstehen, ohne sie zu kritisieren. Mit anderen Worten bedeutet die interkulturelle Kompetenz, ein offenes Weltbild zu haben, indem man Empathie in den Mittelpunkt stellt und mit Menschen aus anderen Kulturen angemessen kommuniziert, indem man sie mit ihren Unterschieden und Ähnlichkeiten akzeptiert. Ebenfalls spielen hier die nonverbalen Phänomene eine wichtige Rolle, und können nicht zur Seite geschoben werden (Yücel, 2009, S. 68). Aufgrund der zunehmenden Internationalisierung sowie Digitalisierung wird die interkulturelle Kompetenz heute als Schlüsselkompetenz verstanden (Genkova und Kruse, 2021, S.138). Interkulturell unkompetente Menschen, die mangelnde Kenntnisse über die Zielkultur haben, können mit negativen Situationen im Zielsprachenland konfrontiert werden. Dies wirkt sich negativ auf die Anpassung der Person an die neue Kultur aus, demzufolge können im Laufe der Zeit aufgrund von Stress negative Auswirkungen im psychischen Gleichgewicht auftreten, wie z. B. geringes Selbstvertrauen, Konzentrationsschwäche, Schlafstörungen, Identitätskrise usw. (Temel, Eğinli, 2011, S. 219).

Um diese negativen Folgen zu vermeiden, im Zielsprachenland frei kommunizieren und kulturelle Unklarheiten verringern zu können, ist es unbedingt notwendig, dass Mitarbeiter internationaler Unternehmen und Personen, die im Ausland studieren, arbeiten oder leben möchten interkulturell kompotent sind. Denn den Fremdsprachenlernenden ermöglicht diese Kompetenz, Einzelheiten der fremdsprachlichen Begebenheiten vor dem Hintergrund ihrer eigenen Sprache und Kultur wahrzunehmen und zu verstehen. Des Weiteren trägt sie dazu bei, Strategien im Umgang mit Neuem, Unvertrautem und Unverständlichem zu erwerben und zu erweitern.

Obwohl für Fremdsprachenlerner, die in zielsprachenfernen und kulturell unterschiedlichen Regionen leben, diese Kompetenz leider nicht sehr leicht zu entwickeln ist, können sie im Zeitalter der Digitalisierung mithilfe von sozialen Medien, z.B. mit einem deutschen Profil bei Instagram oder in einem Video-Kanal bei YouTube etc. die Gelegenheit finden, die Zielkultur zu erleben.

Diese Arbeit geht dieser Hypothese nach und untersucht mit Hilfe von den Studierenden im Studienfach Germanistik an der Universität Selcuk, ob die Fremdsprachenlerner durch soziale Netzwerke landeskundliches Wissen über die Zielkultur sammeln können, und welche Rolle diese Medien beim informellen landeskundlichen Lernen spielen.

\section{Zielsetzung und Methodik}

Ziel dieser Studie ist herauszufinden, ob man durch soziale Medien landeskundliches Wissen über die Zielkultur erwerben kann, und welche Rolle die sozialen Medien beim informellen landeskundlichen Lernen spielen. Um dies herauszufinden, wurde eine quantitative Untersuchung durchgeführt.

Die Datenerhebung erfolgte mittels einer Umfrage, an der die Studierenden im Studienfach Germanistik der Universität Selcuk teilgenommen haben. Die Gretchenfrage in dieser Untersuchung war, ob die Studierenden der Ansicht sind, dass soziale Netzwerke einen positiven Einfluss auf den Erwerb der Zielkultur haben.

Um Antworten auf diese Frage zu finden, wurde ein strukturierter Fragebogen erarbeitet. Die formulierten Fragen thematisierten folgende Aspekte: Allgemeine Angaben zum Geschlecht und Alter der Teilnehmenden, Angaben zur Nutzung der sozialen Medien (wie viel Zeit sie darin verbringen, welche Netzwerke am meisten beliebt sind etc.) und welche Rolle die sozialen Netzwerke beim Erwerb der Zielkultur spielen. Die Befragung erfolgte im Wintersemester 2019-2020, befragt wurden Studierende im Alter ab 18 Jahren. Die Beantwortung des Fragebogens dauerte ca. 5 Minuten. Die Angaben der Studierenden wurden systematisch kodiert und es wurden Häufigkeitsauszählungen vorgenommen. 


\section{Zum Begriff der Interkulturalität und Interkulturelle Kompetenz}

Da unter dem Begriff „Kultur“ „,ie gesamte Lebenswirklichkeit der in einem bestimmten Sprach-und Kulturraum lebenden Menschen, d.h. alle Produkte und Tätigkeiten ibres Denkens und Handelns" (Padrós und Biechele, 2003, S. 12) verstanden wird, ist „Interkulturalität“ nicht als eine soziale Struktur zu verstehen, sondern vielmehr als ein Verlauf, der sich auf das Zusammenleben von Menschen verschiedener Kulturen und auf ihr Verhältnis zueinander bezieht (Bolten, 2012, S. 39). Sie beruht auf Beziehungen zwischen verschiedenen Lebenswelten und ist ein lebendiger Interaktionsprozess, der gegenseitiges Bewusstsein, sowie Respekt und Anerkennung entwickelt. Zwischen den Mitgliedern verschiedener Kulturen entsteht aufgrund dieser Wechselbeziehung die sogenannte Interkultur, die immer neu erzeugt wird (Wille, 2016, S. 54). Um die Angehörigen dieser Kulturen besser verstehen und an ihren Gesprächen problemlos teilnehmen zu können, sollte man über ihre Lebensformen (Handlungsweise, Wahrnehmung, Gefühle, Weltanschauung etc.) etwas wissen.

Doch mit diesem Wissen ist kein explizites Wissen gemeint, das durch Nachschlagen in Fachbüchern erworben wird, sondern ein implizites Wissen, d.h. praktisches Können, das nur in direkten und originalen Erfahrungen mit Angehörigen der Zielkultur erreicht werden kann (Loenhoff, 2014, S. 33). Für Fremdsprachenlerner, die in zielsprachenfernen und kulturell unterschiedlichen Regionen leben ist es aber meistens nicht möglich außer Lehrbücher, die meist keine authentische Elemente enthalten, eine Innenansicht der Zielkultur zu bekommen und auf diese Weise die Einzelheiten der fremdsprachlichen Begebenheiten vor dem Hintergrund ihrer eigenen Sprache und Kultur wahrzunehmen und zu verstehen.

Eine Fremdsprache zu beherrschen bedeutet also nicht nur über ein ausreichendes, sprachliches Wissen zu verfügen, sondern vielmehr in interkulturellen Situationen richtig und angemessen kommunizieren zu können. Demgemäß setzt der Europarat, Rat für kulturelle Zusammenarbeit, im gemeinsamen europäischen Referenzrahmen (2001, S. 103-106) neben den vier Fertigkeiten (Lese- und Hörverstehen, Sprechen und Schreiben) die Interaktionsfähigkeit als fünfte Kompetenz im Sprachgebrauch voraus.

Unter dem Begriff „Kompetenz“ versteht man Fertigkeiten und Einstellungen etc. sowie Kontext- und Erfahrungswissen, die bei Individuen verfügbar oder durch sie erlernbar sind und können in bestimmten Gegebenheiten abgerufen und angeregt werden um bestimmte Probleme zu lösen. Demzufolge ist „Interkulturelle Kompetenz“ die Fähigkeit, effektiv und angemessen in interkulturellen Situationen kommunizieren zu können (Wille, 2016, S. 55). Interkulturelle Kompetenz wird unter anderem auch als eine Empathiefähigkeit verstanden, sich in Menschen aus anderen Kulturen hineinzuversetzen und zu versuchen, ihr Verhalten und ihre Gedanken zu verstehen, ohne sie zu kritisieren.

Weiterhin bedeutet interkulturelle Kompetenz ein offenes Weltbild zu haben, indem man Empathie in den Mittelpunkt stellt und mit Menschen aus anderen Kulturen angemessen kommuniziert, indem man sie mit ihren Unterschieden und Ähnlichkeiten akzeptiert. Den Fremdsprachenlernenden ermöglicht diese Kompetenz, Einzelheiten der fremdsprachlichen Begebenheiten vor dem Hintergrund ihrer eigenen Sprache und Kultur wahrzunehmen und zu verstehen. Außerdem trägt sie dazu bei, Strategien im Umgang mit Neuem, Unvertrautem und Unverständlichem zu erwerben und zu erweitern.

\section{Die Rolle der sozialen Medien beim landeskundlichen Lernen}

Vor allem mit dem Internet hat ein neues Zeitalter begonnen. Die schnelle Verbreitung von Informationen, Fotos, Videos und Symbolen auf den Plattformen wie Facebook, Instagram, Twitter etc. erfüllen somit kommunikative Zwecke (Kneuer und Richter, 2015, S. 15). Nicht nur Lehrbücher sind beim landeskundlichen Lernen die Quelle für landeskundliche Informationen, soziale Medien sind ebenfalls in den Fremdspracheunterricht zur Geltung gekommen, und können bei der Didaktik der Landeskunde gebraucht werden (Padrós und Biechele, 2003, S. 119-120). Unter dem Begriff „Soziale Medien" werden eine Menge verschiedener Web-Angebote verstanden, wie z.B. Instagram, Facebook, YouTube, Twitter usw. Hier handelt es sich um eine Vielfalt von digitaler Netzwerke und Technologien, 
mit deren Hilfe die Nutzer Informationen gegenseitig austauschen und mediale Themen alleine oder in Gemeinschaft darstellen können.

Mit der gegenseitigen Übertragung von Informationen, Ansichten, Stellungnahmen usw. ist sie gleichzeitig auch eine Interaktion, weil die Nutzer anhand von Kommentaren oder Bewertungen auf die Inhalte zurückgreifen, und damit einen sozialen Kontakt aufbauen. Die Interaktionsform kann Text, Ton, Bild, Video oder ein einfacher Klick sein (Scheffler, 2014, S. 13-14). Mit Menschen verschiedener Identitäten und Glaubensrichtungen tragen die sozialen Medien auch zur Globalisierung bei.

Da im Mittelpunkt landeskundlichen Lernens die Menschen des Zielsprachenlandes stehen und im Fremdsprachenunterricht vor allem ihr Alltag gezeigt werden soll (Padrós und Biechele, 2003, S. 8), dienen die Stories oder Posts der Muttersprachler des Zielsprachenlandes auf Instagram, Facebook usw. dazu die Alltagskultur auf authentische Weise zu widerspiegeln und auf diese Weise einen Einblick in die fremde Welt zu gewinnen. Aus diesem Grund können sie auch als authentische, gesprochene Texte gesehen werden, die in der Realität existieren.

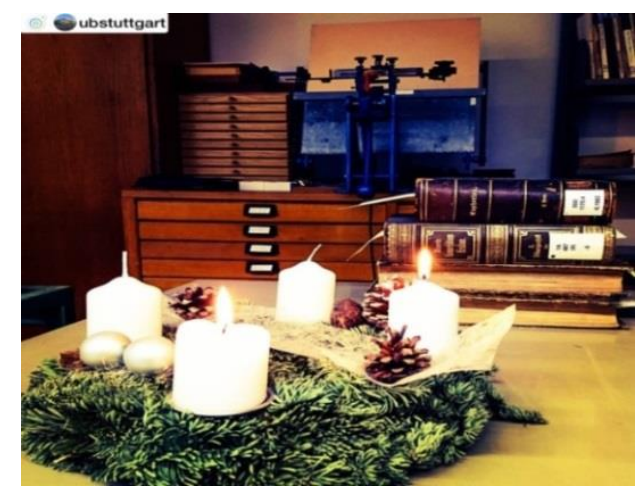

Bild 1. Ein Bild von einem Adventskranz aus dem offizielen Instagram-Account der Universitätsbibliothek Stuttgart in der Adventszeit. Quelle: https://www.instagram.com/ubstuttgart/

Bild 1 von einem Adventskranz aus dem offizielen Instagram-Account der Universitätsbibliothek Stuttgart zeigt, wie ein Adventskranz aussieht, und wann die Kerzen in der Adventszeit angezündet werden. Solche Bilder dienen nicht nur dazu, einen Einblick in die fremdsprachliche Kultur zu geben, sondern auch die Motivation und die Neugier der Lernenden auf die Zielkultur zu wecken.

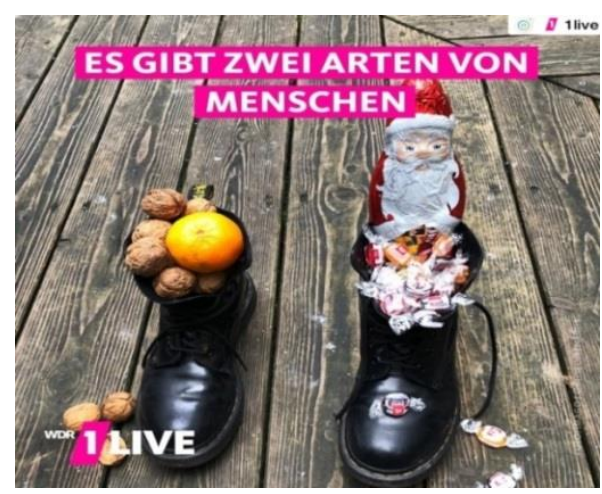

Bild 2. Ein Bild aus dem Instagram-Account des deutschen Webradios 1Live am Nikolaustag

Quelle: https://www.instagram.com/1live/ 


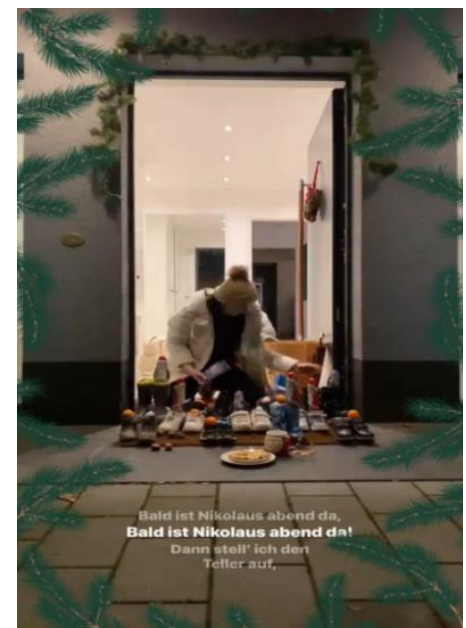

Bild 3. Ein Bild aus der Instagram-Story einer deutschen Muttersprachlerin am Nikolaustag

Quelle: https://www.instagram.com/die.kim/

Bild 2 aus dem Instagram-Account des deutschen Webradios 1Live, und Bild 3 aus der InstagramStory der deutschen Muttersprachlerin die.kim am Nikolaustag zeigen, wie und wann die Nikolausstiefel am Nikolaustag in Deutschland gefüllt werden. Die Fremdsprachenlerner können mithilfe dieser zahlreichen Bilder und Videos in den sozialen Medien die Gelegenheit finden, die Zielkultur zu erleben.

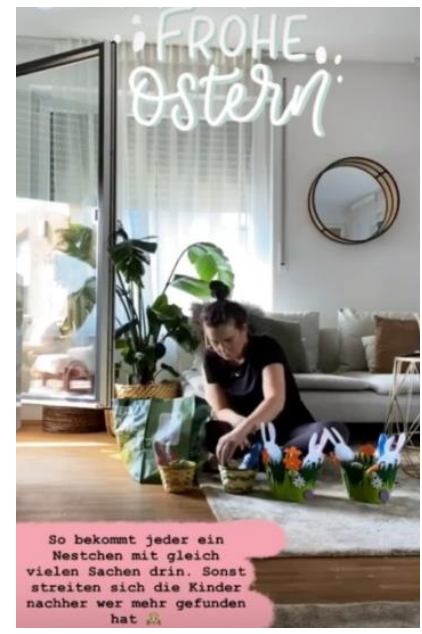

Bild 4. Ein Bild aus der Instagram-Story einer deutschen Muttersprachlerin an Ostern

Quelle: https://www.instagram.com/die.kim/ 


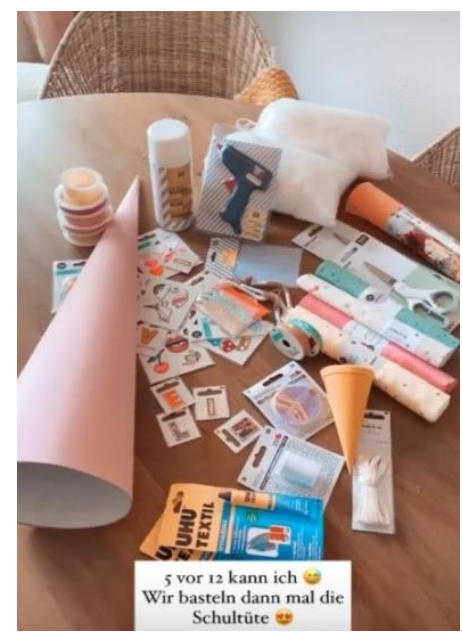

Bild 5. Ein Bild aus der Instagram-Story einer deutschen Muttersprachlerin vor dem Schulanfang

Quelle: https://www.instagram.com/die.kim/

Bild 4 und 5 sind auch einige Beispiele aus den Instagram-Stories von einer deutschen Muttersprachlerin, die landeskundliches Wissen über die Zielkultur geben. Beispielsweise wird in Bild 4 Osterneste gefüllt, in derselben Instagram-Story wird auch gezeigt, wie ein Frühstückstisch an Ostern eingedeckt wird, und wie die Deutschen das Osterfest feiern.

Weiterhin zeigt Bild 5, wie eine Schultüte gebastelt wird. Fremdsprachenlerner können mithilfe von dieser authentischen Instagram-Story auch erfahren, was eine Schultüte ist und wozu sie gebraucht wird. Hier ist es von großer Bedeutung, dass die Gespräche nicht künstlich gesteuert sind, und eine Innenansicht der Zielkultur geben. Diese Bilder sind nur einige Beispiele, die den Lernenden der Zielsprache landeskundliches Wissen über die Zielkultur geben.

Ob soziale Netzwerke wirklich zum landeskundlichen Lernen beitragen, und welche Rolle sie beim landeskundlichen Lernen spielen, wird im folgenden Teil dieses Beitrags durch die Ergebnisse einer Umfrage wiedergegeben.

\section{Umfrageergebnisse}

In diesem Teil werden die Ergebnisse einer Umfrage vorgestellt, an der etwa 150 Studierende im Studienfach Germanistik der Universität Selcuk teilgenommen haben. Das Ziel dieser Umfrage ist herauszufinden, ob die Studierenden durch soziale Medien landeskundliches Wissen über die Zielkultur erwerben können und welche Rolle die sozialen Medien beim informellen landeskundlichen Lernen spielen.

Um Antworten auf diese Fragen zu finden wurde ein Fragebogen erarbeitet, die formulierten Fragen thematisierten folgende Aspekte: Allgemeine Angaben zum Geschlecht und Alter der Teilnehmenden, Angaben zur Nutzung der sozialen Medien (wie viel Zeit sie darin verbringen, welche Netzwerke am meisten beliebt sind etc.) und welche Rolle die sozialen Netzwerke beim Erwerb der Zielkultur spielen.

Die Befragten wurden zunächst gebeten anzugeben, ob sie soziale Netzwerke nutzen. $92 \%$ der Befragten haben diese Frage mit „Ja“ beantwortet, nur 8\% mit „Nein“. Die folgende Abbildung 1 stellt dar, dass fast alle der an der Umfrage teilnehmenden Studierenden, soziale Netzwerke nutzen. 


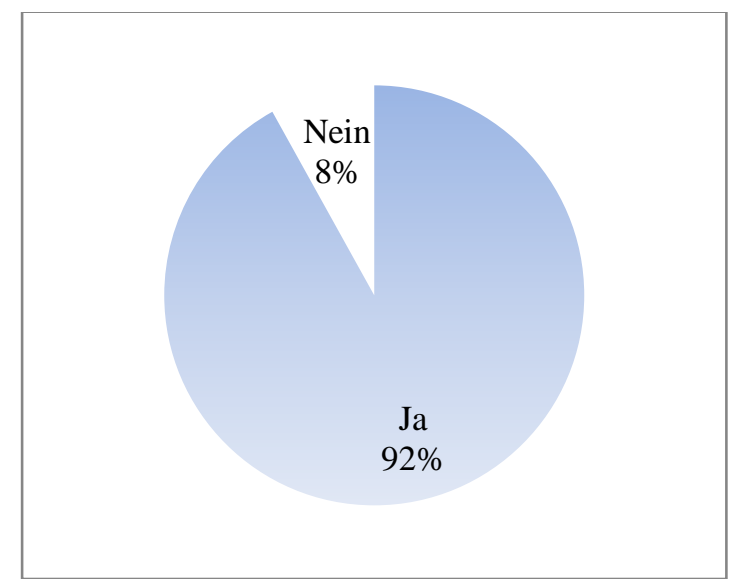

Abbildung 1. Nutzung sozialer Netzwerke

Auf die Frage, wie viel Zeit sie in den sozialen Netzwerken verbringen, antworteten die Teilnehmer unterschiedlich. Die überwiegende Mehrheit der Befragten gab an, dass sie täglich 1-3 Stunden Zeit in sozialen Netzwerken verbringen, wie auch aus Abbildung 2 ersichtlich ist.

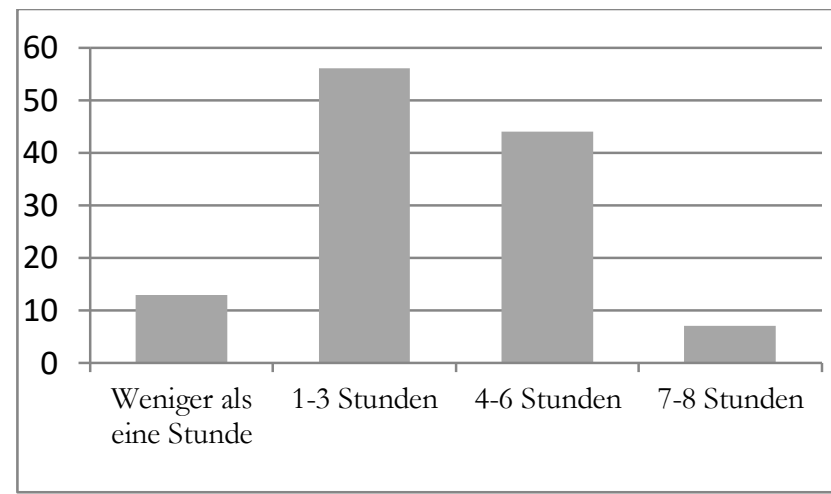

Abbildung 2. Durchschnittliche Zeit in sozialen Netzwerken

Die nachfolgende Abbildung 3 gibt die Angaben der befragten Studierenden nach den beliebtesten sozialen Netzwerken an. Ein relativ hoher Anteil der Befragten gab an, dass die beliebtesten und häufig genutzten sozialen Netzwerke Instagram und die Videoplattform YouTube sind.

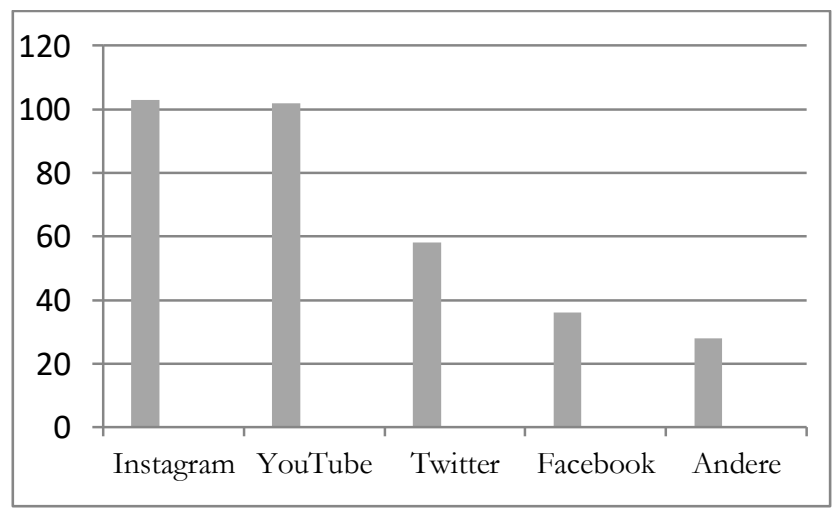

Abbildung 3. Angaben der befragten Studierenden zur Frage nach den beliebtesten sozialen Netzwerken 
Bei der Umfrage wurden die Studierenden außerdem danach gefragt, ob sie durch soziale Netzwerke landeskundliches Wissen über die Zielkultur erwerben bzw. die Zielkultur kennenlernen können. Das Ergebnis wird in Abbildung 4 wiedergegeben.

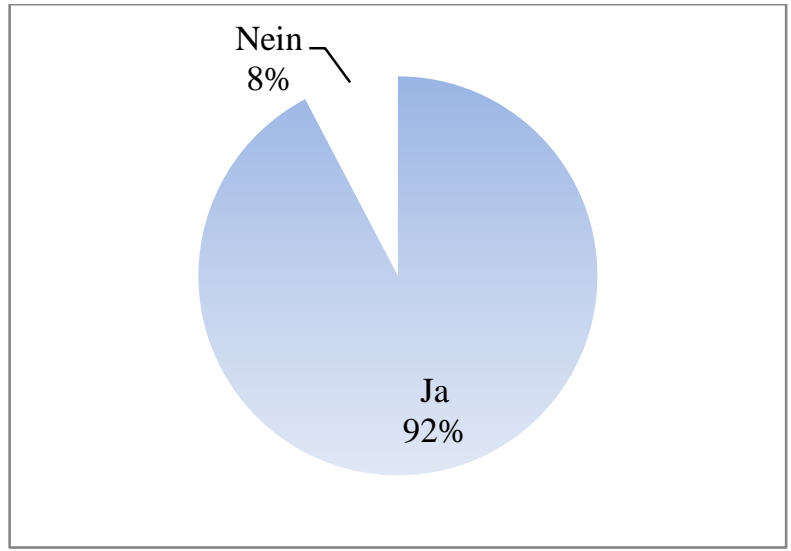

Abbildung 4. Angaben der befragten Studierenden zur Frage nach der Rolle der sozialen Medien beim Kennenlernen der Zielkultur

Abbildung 1 und 4 deuten darauf hin, dass alle befragten Studierenden, die soziale Netzwerke nutzen, landeskundliches Wissen über die Zielkultur erwerben und damit auch die Zielkultur kennenlernen können.

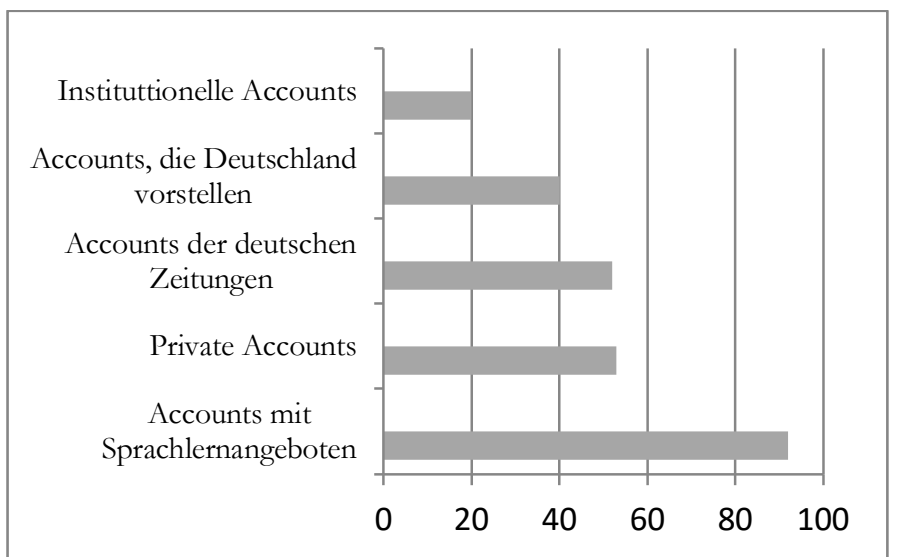

Abbildung 5. Angaben zu den gefolgten Seiten in sozialen Netzwerken

In der Umfrage wurden die Studierenden außerdem danach gefragt, welchen deutschsprachigen Seiten sie in sozialen Netzwerken folgen. Die Befragten gaben an, dass sie am meisten Accounts mit Sprachlernangeboten folgen. Weitere Aussagen befinden sich in Abbildung 5. 


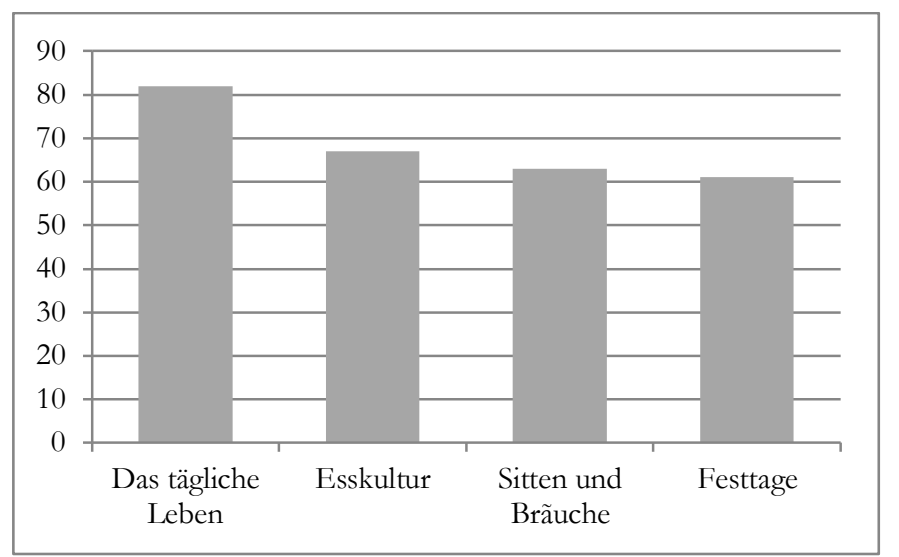

Abbildung 6. Angaben zum Erwerb landeskundliches Wissen in sozialen Netzwerken

Die befragten Studierenden wurden gebeten anzugeben, was sie bisher in sozialen Netzwerken über die Zielkultur gelernt haben. Wie aus Abbildung 6 ersichtlich, geben die große Mehrheit der Studierenden an, dass sie in sozialen Netzwerken am meisten über das tägliche Leben des Zielsprachenlandes etwas lernen. Die Esskultur, Bräuche und Festtage des Zielsprachenlandes sind weitere kulturelle Gegebenheiten, die die Studierenden in sozialen Medien über die Zielkultur erwerben. Anhand der vorliegenden Studie konnte auch aufgezeigt werden, dass ein Großteil der befragten Studierenden in sozialen Netzwerken Accounts über die Zielsprache und Zielkultur folgen.

Die Umfrageergebnisse deuten darauf hin, dass in dem Zeitalter der Digitalisierung die Nutzung sozialer Netzwerke sehr verbreitet ist und, dass die befragten Studierenden viel Zeit damit verbringen, anhand der vorliegenden Studie konnte auch aufgezeigt werden, dass die soziale-Netzwerk-Plattform Instagram bei den Studierenden besonders beliebt ist.

Im Gesamten betrachtet, bestätigt die vorliegende Umfrage, dass die sozialen Netzwerke einen positiven Einfluss auf den Erwerb der interkulturellen Kompetenz haben.

\section{Schlussfolgerungen}

Wir befinden uns im digitalen Zeitalter, derzeit hat fast jeder Zugang zum Internet und verbringt mehrere Stunden pro Tag auf sozialen Netzwerken. Dies hat nicht nur negative, sondern auch positive Auswirkungen: Beispielsweise können die Fremdsprachenlerner, die in zielsprachenfernen Regionen leben, mit Hilfe von sozialen Netzwerken eine Innenansicht der Zielkultur bekommen, indem sie YouTube-Kanäle oder Instagram-Accounts folgen, die die Zielkultur explizit oder implizit reflektieren.

Lehrbücher und Lehrkräfte sind nicht mehr die einzige Quelle um landeskundliches Wissen über die Zielkultur zu sammeln. Da in den Lehrbüchern meistens nicht authentische, sondern eher künstlich gesteuerte Gespräche behandelt werden, sind die Stories, Videos oder Posts der Muttersprachler des Zielsprachenlandes auf Instagram, YouTube, Facebook usw. mehr dazu geeignet, die Alltagskultur auf authentische Weise zu widerspiegeln und auf diese Weise einen Einblick in die fremde Welt zu gewinnen. Wegen ihrer Authentizität geben sie den Fremdsprachenlernern die Möglichkeit, Zugang zu anderen Kulturen zu finden, was ein übergeordnetes Ziel des Fremdsprachenunterrichts ist und dienen auch dazu die Zielkultur bewusst oder unbewusst fast hautnah zu erleben.

In diesem Beitrag wurde dieses Thema näher bearbeitet. Zunächst wurden die Begriffe Interkulturalität und interkulturelle Kompetenz in die Hand genommen, danach wurde die Rolle der sozialen Medien beim landeskundlichen Lernen anhand von einigen Beispielen untersucht, und schließlich wurden die Ergebnisse einer Umfrage präsentiert sowie interpretiert, an der die Studierenden im Studienfach Germanistik der Universität Selcuk teilgenommen haben. 
Ziel dieser Studie war festzustellen, ob Fremdsprachenlerner durch soziale Medien landeskundliches Wissen über die Zielkultur erwerben können, und welche Rolle die sozialen Medien beim informellen landeskundlichen Lernen spielen. Am Ende der Untersuchung stellte es sich heraus, dass die befragten Studierenden, die soziale Netzwerke nutzen, mit Hilfe dieser Mitteln landeskundliches Wissen über die Zielkultur erwerben und damit auch die Zielkultur kennenlernen können.

Stories oder Posts der Muttersprachler des Zielsprachenlandes auf soziale Netzwerke, wie Instagram, Facebook, Twitter, YouTube etc. dienen also dazu, die Alltagskultur auf authentische Weise zu widerspiegeln, und auf diese Weise die Menschen hinter dieser Kultur genauer kennenlernen und letztendlich auch verstehen zu können. Hiermit können außerdem Einzelheiten der fremdsprachlichen Begebenheiten vor dem Hintergrund der eigenen Sprache und Kultur wahrgenommen und verstanden werden. Was für den Fremdsprachenlerner, die in zielsprachenfernen und kulturell unterschiedlichen Regionen leben, einen wichtigen Stellenwert hat und zur Entwicklung interkultureller Kompetenz als übergeordnetes Ziel interkultureller Bildung beiträgt.

\section{Extended Abstract}

Foreign language didactics has been influenced by its related sciences since it existed as a science. These and other disciplines have left their mark on all foreign language didactic models, such as the direct method, the audiovisual approach, the cognitive approach or the new intercultural didactics. If we take a closer look at the development of foreign language didactics in the last twenty years, it is found that intercultural didactics, the aim of which is to acquire intercultural competence, is gaining in importance.

Intercultural competence is understood, as the ability to empathize with people from other cultures and to try to understand their behavior and thoughts without criticizing them. In other words, intercultural competence means having an open worldview by focusing on empathy and communicating appropriately with people from other cultures by accepting them with their differences and similarities. This competence enables foreign language learners to perceive and understand details of foreign language events against the background of their own language and culture. It also helps to acquire and expand strategies for dealing with the new, the unfamiliar and the incomprehensible. In order to be able to communicate correctly and appropriately in intercultural situations, intercultural competence plays a very important role. For this reason, the Council of Europe, Council for Cultural Cooperation, in the Common European Framework of Reference assumes the ability to interact as a fifth competence in language use in addition to the four skills "reading and listening comprehension, speaking and writing. But for foreign language learners who live in regions that are far from the target language and culturally different, it is usually not possible to get an inside view of the target culture, apart from textbooks, which usually contain no authentic elements, and in this way the details of the foreign language occurrences against the background of their own language and culture perceive and understand.

In the digital age has almost everyone access to the Internet and spends several hours a day on social media. This has not only negative but also positive effects: For example, foreign language learners who live in regions remote from the target language can use social media to get an inside view of the target culture by following YouTube channels or Instagram accounts that explicitly or implicitly describe the target culture reflect. So textbooks are no longer the only source for collecting regional knowledge about the target culture. Since the textbooks mostly do not deal with authentic, but rather artificially controlled conversations, the stories, videos or posts by the native speakers of the target language country on Instagram, YouTube, Facebook, etc. are more suitable for reflecting everyday culture in an authentic way and in this way to gain an insight into the foreign world.

The aim of this study is to find out with the help of around 150 German studies students at the University of Selcuk, examines whether foreign language learners can use social networks to collect knowledge about the target culture and what role these media play in learning about the country. To find answers to these questions, a questionnaire was drawn up. The questions formulated addressed the 
following aspects: general information on the gender and age of the participants, information on the use of social media (how much time they spend in it, which networks are most popular, etc.) and what role the social networks play in acquiring the target culture. The survey results indicate that in an age of digitization, the use of social networks is very widespread and that the surveyed students spend a lot of time with them. It turned out that the surveyed students who use social networks acquire regional knowledge about the target culture and thus also get to know the target culture. Viewed as a whole, the present survey confirms that social networks have a positive influence on the acquisition of intercultural competence.

Keywords: Culture, Interculturality, Intercultural Competence, Regional Studies, Social Media.

\section{Literaturverzeichnis}

Bolten, J. (2012). Interkulturelle Kompeten₹: Thüringen: Landeszentrale für politische Bildung.

Europarat - Rat für kulturelle Zusammenarbeit (2001). Gemeinsamer Europäischer Referenærahmen für Sprachen: lernen, lebren, beurteilen. Berlin: Langenscheidt.

Genkova, P. ; Kruse L. (2021). Auslandsaufenthalt als Kompetenzschule? Eine empirische Untersuchung der Auswirkungen von Auslandsaufenthalten auf die berufsbezogenen Kompetenzbereiche. In: Gruppe. Interaktion. Organisation. Wiesbaden: Springer.

Kneuer, M. ; Richter S. (2015). Soziale Medien in Protesbewegungen. Neue Wege für Diskurs, Organisation und Empörung? Frankfurt: Campus Verlag.

Krumm, H. J. (1994). Zur Situation der Lehrwerkkritik und Lehrwerkforschung Deutsch als Fremdsprache. In: Zur Analyse, Begutacbtung und Entwicklung von Lebrwerken, (Hrsg: Bernd Kast und Gerhard Neuner). Berlin: Langenscheidt.

Loenhoff, J. (2014). Kulturelle Differenz, interkulturelle Kommunikation und die Funktion impliziten Wissens. In: Dialog und (Inter-)Kulturalität. Theorien, Konzepte, empirische Befunde. (Hrsg.: S. Meier, D. H. Rellstab, G. L. Schiewer). Tübingen: Narr Francke Attempto Verlag.

Padrós, A. ; Markus, B. (2003). Didaktik der Landeskunde. (Hrsg. Gerhard Neuner). Berlin: Druckhaus Langenscheidt.

Reimann, D. (2015). Inter- und transkulturelle kommunikative Kompeten₹: ProDaæ: Deutsch als Zweitsprache in allen Fächern. Universität Duisburg, Fakultät für Geisteswissenschaften, Institut für Deutsch als Zweit- und Fremdsprache.https://www.unidue.de/imperia/md/content/prodaz/reimann_intertranskulturelle_komp etenz.pdf. Zugriff am: 15.06.2021.

Scheffler, H. (2014). Soziale Medien - Einführung in das Thema aus Sicht der Marktforschung. In: Soziale Medien - Gegenstand und Instrument der Forschung. (Hrsg.: Ch. König, M. Stahl, E. Wiegand). Wiesbaden: Springer.

Temel, Eğinli A. (2011). Kültürlerarası Yeterlilï̆in Kazanılmasinda Kültürel Farklllk. ve Eğitimlerinin Önemi. In: Öneri Dergisi. https://dergipark.org.tr/en/pub/maruoneri/issue/17898/187793. [Zugriff am: 13.06.2021]

Wille, K. (2016). Interkulturelle Kompetenz als übergreifendes Ziel in der schulischen Bildung. In: International Dialogues on Education: Past and Present. 3, (1). 52-69.

Winkler, O. (2014). Dialog und Kulturalität in der historischen Dialogforschung - Überlegungen am Beispiel von Dramentexten. In: Dialog und (Inter-)Kulturalität. Theorien, Konzepte, empirische Befunde. (Hrsg.: S. Meier, D. H. Rellstab, G. L. Schiewer). Tübingen: Narr Francke Attempto Verlag.

Yücel, Erdinç (2009). Eine Kulturbrücke für den Fremdsprachenlerner. Konya: Çizgi Kitabevi.

Bild 1: https://www.instagram.com/ubstuttgart/ [Zugriff am: 13.12.2020].

Bild 2: https://www.instagram.com/1live/ [Zugriff am: 06.12.2020].

Bild 3: https://www.instagram.com/die.kim/ [Zugriff am: 06.12.2020].

Bild 4: https://www.instagram.com/die.kim/ [Zugriff am: 11.04.2020]. 
Bild 5: https://www.instagram.com/die.kim/ [Zugriff am: 28.08.2020]. 